\title{
The presence of the ancestral insect telomeric motif in kissing bugs (Triatominae) rules out the hypothesis of its loss in evolutionarily advanced Heteroptera (Cimicomorpha)
}

\author{
Sebastián Pita', Francisco Panzera', Pablo Mora², Jesús Vela², \\ Teresa Palomeque ${ }^{2}$, Pedro Lorite ${ }^{2}$
}

I Sección Genética Evolutiva, Facultad de Ciencias, Universidad de la República, Montevideo, Uruguay 2 Departamento de Biología Experimental, Área de Genética, Universidad de Jaén, Jaen, Spain

Corresponding author: Pedro Lorite (plorite@ujaen.es)

Academic editor: Seppo Nokkala | Received 21 July 2016 | Accepted 22 August 2016 | Published 13 September 2016

http://zoobank.org/63D07517-625E-430A-B75F-9854DA8C4179

Citation: Pita S, Panzera F, Mora P, Vela J, Palomeque T, Lorite P (2016) The presence of the ancestral insect telomeric motif in kissing bugs (Triatominae) rules out the hypothesis of its loss in evolutionarily advanced Heteroptera (Cimicomorpha). Comparative Cytogenetics 10(3): 427-437. doi: 10.3897/CompCytogen.v10i3.9960

\begin{abstract}
Next-generation sequencing data analysis on Triatoma infestans Klug, 1834 (Heteroptera, Cimicomorpha, Reduviidae) revealed the presence of the ancestral insect (TTAGG) telomeric motif in its genome. Fluorescence in situ hybridization confirms that chromosomes bear this telomeric sequence in their chromosomal ends. Furthermore, motif amount estimation was about $0.03 \%$ of the total genome, so that the average telomere length in each chromosomal end is almost $18 \mathrm{~kb}$ long. We also detected the presence of (TTAGG) ${ }_{n}$ telomeric repeat in mitotic and meiotic chromosomes in other three species of Triatominae: Triatoma dimidiata Latreille, 1811, Dipetalogaster maxima Uhler, 1894, and Rhodnius prolixus Ståhl, 1859. This is the first report of the (TTAGG) ${ }_{n}$ telomeric repeat in the infraorder Cimicomorpha, contradicting the currently accepted hypothesis that evolutionarily recent heteropterans lack this ancestral insect telomeric sequence.
\end{abstract}

\section{Keywords}

Cimicomorpha, kissing bugs, holocentric chromosomes, telomeres, NGS, (TTAGG) ${ }_{\mathrm{n}}$ 


\section{Introduction}

Telomeres, the physical ends of eukaryote chromosomes, are defined as specialized DNA-protein structures essential for chromosome replication, meiotic pairing and chromosome stability. In most organisms, telomeric DNA is composed by simple G-rich sequences repeats that extend for tens of base pairs (bp) as much as $150 \mathrm{~kb}$, depending on the organism. Although telomeric repeats are diverse in their DNA sequence composition among different organisms (Zakian 1995), several taxonomic groups possess highly conserved motifs. Vertebrates, including bony fishes, reptiles, amphibians, and mammals exhibit the (TTAGGG) ${ }_{n}$ repeat (Meyne et al. 1989) while the (TTTAGGG) ${ }_{\mathrm{n}}$ sequence appears highly conserved in the plant kingdom (Watson and Riha 2010). Extensive studies in arthropods have revealed that the predominant telomeric sequence is a pentanucleotide sequence repeat (TTAGG), which has been considered as the ancestral telomeric motif in phylum Arthropoda, including insects (Sahara et al. 1999, Frydrychová et al. 2004, Vítková et al. 2005). However, numerous studies contradict this claim. For example several insect groups do not exhibit this telomeric repeat, such as Diptera, Ephemeroptera, Odonata, Dermaptera, Siphonaptera, Mecoptera, Raphidioptera and parasitic Hymenoptera. In addition, Coleoptera, Neuroptera and Hemiptera orders include species with and without the ancestral (TTAGG) telomeric motif (Frydrychová et al. 2004, Gokhman et al. 2014, Korandová et al. 2014). In these insect groups, the ancestral telomeric motif is replaced by other alternative telomeric sequences such as (TCAGG) ${ }_{\mathrm{n}}$ in some coleopteran species (Mravinac et al. 2011), non-long terminal repeat (LTR) retrotransposons in Drosophila Fallén, 1823 (Mason et al. 2008), arrays of long satellite repeats in Culicomorpha dipteran (Walter et al. 2001), or by unknown sequences as in damselflies, mayflies and some aphid species (Frydrychová et al. 2004, Vítková et al. 2005). The most illustrative example of the variability of the telomeric sequences was observed in Coleoptera where ancestral (TTAGG) ${ }_{n}$ has been lost at least eight times during the evolution of this insect group (Frydrychová and Marec 2002, Mravinac et al. 2011).

Among Hemiptera, the ancestral motif is present in the suborder Sternorrhyncha (coccids and aphids with some exceptions) (Mohan et al. 2011, Monti et al. 2011, Novotná et al. 2011), in several genera of Auchenorrhyncha (Frydrychová et al. 2004, Maryańska-Nadachowska et al. 2013, Golub et al. 2014, Kuznetsova et al. 2015a) and Coleorrhyncha (Kuznetsova et al. 2015b) suborders. In the suborder Heteroptera, only two species of the basal infraorders Nepomorpha and Gerromorpha show the ancestral telomeric motif (Kuznetsova et al. 2012, Mason et al. 2016). On the contrary, the most derived and specious heteropteran infraorders (Cimicomorpha and Pentatomomorpha) do not show the classic insect motif (for review see Grozeva et al. 2015, Mason et al. 2016). A recent survey of several sequenced genomes of these groups, including the triatomine Rhodnius prolixus, confirms the lack of the ancestral telomeric repeat and these groups are regarded as having a defective version of telomerase gene (Mason et al. 2016). Mason et al. (2016) have suggested the occurrence of a single loss event of the telomeric repeat, sometime before the Cimicomorpha and Pentatomomorpha divergence, and after their separation from Nepomorpha. 
Kissing bugs (Triatominae, Reduviidae) are included within the infraorder Cimicomorpha (Heteroptera), constituting a group of medical relevance because they act as vectors of Chagas disease, also known as American trypanosomiasis. This subfamily includes 150 species, of which more than 80 have been cytogenetically studied (Panzera et al. 2010), having holocentric chromosomes. The current data, as above mentioned, suggest a high heterogeneity in insect telomere composition. One should also take into consideration that loss of the insect ancestral repeat in Cimicomorpha has been reported (Mason et al. 2016). For all these reasons it is important to explore for the first time in Triatominae the presence of (TTAGG) ${ }_{n}$ motif, using next-generation sequencing (NGS) analysis tools and fluorescence in situ hybridization (FISH) in four triatomine species from three different genera. The results presented in this paper are in clear contradiction to the loss of ancestral telomeric repeats hypothesis in evolutionarily advanced Heteroptera.

\section{Materials and methods}

\section{Material}

Four species where analyzed, involving three different genera from the two principal tribes of the subfamily: Triatomini (Dipetalogaster maxima, Triatoma infestans, and T. dimidiata) and Rhodniini (Rhodnius prolixus). The last three species are the main vectors of Chagas disease. Origin and cytogenetic traits of each species are detailed in Table 1.

\section{Telomere detection by genome sequencing}

A Triatoma infestans (non-Andean lineage) specimen collected in Tacuarembó (Uruguay) was used for sequencing. Approximately $3 \mu \mathrm{g}$ of genomic DNA were employed in a low coverage Illumina ${ }^{\oplus}$ Hiseq $^{\mathrm{TM}} 2000$ paired-end sequencing. Graph-based clustering analysis was carried out using RepeatExplorer (Novák et al. 2013), implemented within the Galaxy environment (http://repeatexplorer.umbr.cas.cz/) (Novák et al. 2010). RepeatExplorer also allow quantifying the abundance of the repeated sequences in the genome in base to the number of reads in each cluster.

\section{Telomere detection by FISH}

Chromosome preparations for FISH analyses were obtained from male gonads. Testes were removed from live adult insects, fixed in an ethanol-glacial acetic acid mixture (3:1) and stored at $-20^{\circ} \mathrm{C}$. Squashes were made in a $50 \%$ acetic acid drop, coverslips were removed after freezing in liquid nitrogen and the slides were air dried and then stored a $4^{\circ} \mathrm{C}$. 
Table I. Geographical origin and male diploid chromosome number in the four species here analyzed. $\mathrm{A}=$ autosomes.

\begin{tabular}{l|c|c}
\hline \multicolumn{1}{c|}{ Species } & Geographical origin & $\begin{array}{c}\text { Male diploid chromosome } \\
\text { number (2n) }\end{array}$ \\
\hline Tribe Rhodniini & \multicolumn{2}{|c}{} \\
\hline Rhodnius prolixus & Guatemala, Quezaltenango, Insectary CDC (USA) & $22=20 \mathrm{~A}+\mathrm{XY}$ \\
\hline Tribe Triatomini & Baja California, Mexico \\
\hline Dipetalogaster maxima & Jutiapa, Guatemala & $22=20 \mathrm{~A}+\mathrm{XY}$ \\
\hline Triatoma dimidiata & Tacuarembó, Uruguay & $22=20 \mathrm{~A}+\mathrm{X}_{1} \mathrm{X}_{2} \mathrm{XY}$ \\
\hline Triatoma infestans &
\end{tabular}

Telomeric TTAGG probe generation and FISH assays were carried out following Lorite et al. (2002) and Mora et al. (2015). Telomeric probes were generated by PCR using the primers (TTAGG) ${ }_{6}$ and (TAACC), following a similar procedure as described by IJdo et al. (1991). PCR was performed in $100 \mu \mathrm{l}$ using $100 \mathrm{pmol}$ of each primer and 2.5 units of Taq polymerase, in the absence of a template. PCRs were carried out using the following cycling profile: 30 cycles at $95^{\circ} \mathrm{C}(60 \mathrm{sec}), 50^{\circ} \mathrm{C}(1 \mathrm{~min}), 72^{\circ} \mathrm{C}(3 \mathrm{~min})$, with a final elongation step of $72^{\circ} \mathrm{C}$ for $10 \mathrm{~min}$. PCR generated fragments (between $200 \mathrm{bp}$ and $1 \mathrm{~kb}$ ) were purified and labeled with biotin-16-dUTP (Roche) out using the Nick Translation Kit (Roche), following manufacturer's instructions. The labelled probe was precipitated and dissolved in $50 \%$ formamide.

Previously to hybridization, slides were treated with RNase A, pepsin and formaldehyde and dehydrated in 70\%, 90\% and 100\% ethanol for 5 min each. Hybridization was performed applying $25 \mu \mathrm{l}$ of DNA labelled solution to each slide, which was heated for $3 \mathrm{~min}$ at $80^{\circ} \mathrm{C}$ to denature the DNA, and immediately chilled on ice for $3 \mathrm{~min}$. The hybridization mix consisted of (final concentrations) 50\% formamide, 2xSSC, $50 \mathrm{mM}$ sodium phosphate, $0.1 \mathrm{mg} / \mathrm{ml}$ sonicated salmon sperm DNA, $0.1 \mathrm{mg} / \mathrm{ml}$ yeast RNA, and $5 \mathrm{ng} / \mathrm{ml}$ labeled telomere probe. The slides were transferred to a moist chamber humidified with formamide $(50 \%)$ and incubated overnight at $37^{\circ} \mathrm{C}$. After incubation, the slides were washed in $50 \%$ formamide at $37^{\circ} \mathrm{C}$, three times, 3 min each; followed by 2xSSC, $0.05 \%$ Tween-20, pH 7.5, three times, 5 min each. Fluorescence immunological detection was performed using the avidin-FICT/ anti-avidin-biotin system with four rounds of amplification. Slides were mounted with Vectashield (Vector). DAPI in the antifade solution was used to counterstain chromosomes.

\section{Results and discussion}

The data obtained from the $T$. infestans genome sequencing were analyzed with RepeatExplorer (Novák et al. 2013). One of the obtained clusters was formed by a telomeric sequence TTAGG array. In order to test if this repeat represents the putative telomere, FISH was carried out using the TTAGG repeat as probe. Hybridization signals were clearly seen at the ends of the mitotic chromosomes (Fig. 1A), revealing that telomeres in this species are really composed by this ancestral insect motif. The cluster of 
the (TTAGG) ${ }_{n}$ sequences was estimated for about $0.0266 \%$ of the total genome size, i.e. $395.5 \mathrm{~kb}$. Considering that the haploid genome content in T. infestans is $1.52 \mathrm{pg}$ $(1.487 \mathrm{Mb}$ ) (Panzera et al. 2007, 2010) and that the chromosome number is $2 \mathrm{n}=22$, the average telomeres length motifs in each chromosome end would be almost $18 \mathrm{~kb}$ long. This value is in the range of the telomere length observed in other insects with the ancestral motif or a variant of this repeat such as Tenebrio molitor Linnaeus, 1758 (15 kb) (Richards et al. 2008) but higher than the observed in other species with holocentric chromosomes as lepidopteran species (6-9 kb) (Okazaki et al. 1993, Mandrioli 2002), or in the homopteran coccid Planococcus lilacinus Cockerell, 1905 (6.4 kb) (Mohan et al. 2011).

Furthermore, we tested the telomeric motif presence by FISH in other three triatomine species with (TTAGG) ${ }_{n}$ probe. Hybridization signals were clearly seen on the chromosomal ends of mitotic and meiotic chromosomes (Fig. 1B-D), revealing that Triatominae telomeres are composed by the ancestral insect motif. FISH technique in triatomines is highly sensitive to material fixation conditions. Cytoplasmic remnants in the slides represent the greatest challenge because it hinders the access of the telomeric probes to the chromosomes. This can be partially avoided using recently extracted gonads. In addition, access of the telomeric probes to the chromosome and its visualization are very sensitive to the chromosomes being on the same plane. As a result, differences in hybridization signals can be observed in the same slide or even within chromosomes of the same cell (Fig. 1).

Given our positive FISH hybridization results on $R$. prolixus chromosomes, we additionally conducted a BLAST search of telomeric sequences in the published genome of this species, available at https://www.vectorbase.org/. Similar as reported by Mason et al. (2016), we did not find (TTAGG) repeats, so that these tandem sequences and probably others repeated sequences are not included in the published genome of $R$. prolixus (Mesquita et al. 2015). This reveals the difficulty of the repetitive DNA fraction assembly, as has been reported in different organisms including the well-studied human genome, making that many repetitive sequences have been omitted from the reference assembly and from most genome-wide analyses (Altemose et al. 2014).

Heteroptera or true bugs are a hemipteran suborder comprising seven infraorders and 40,000 species. All phylogenetic studies agreed that the infraorders Cimicomorpha and Pentatomomorpha are the most evolutionarily derived groups, with a common ancestor and involving about $80 \%$ of heteropteran species (Weirauch and Schuh 2011). Until now, the detection by FISH, Southern and/or dot-blot hybridization of telomeric repeat motif (TTAGG) ${ }_{n}$ in Heteroptera has been unsuccessful in nine genera from five families of the infraorders Cimicomorpha and Pentatomomorpha (Sahara et al. 1999, Kuznetsova et al. 2011, Frydrychová et al. 2004, Grozeva et al. 2011, Golub et al. 2015). Only two heteropteran species from the basal infraorders Nepomorpha and Gerromorpha exhibit the ancestral telomeric motif (Kuznetsova et al. 2012, Mason et al. 2016). The (TTAGG) motif was suggested to be lost in the early evolution being and secondarily replaced by another motif or an alternative telomerase-independent mechanism of telomere maintenance (Frydrychová et al. 2004, Lukhtanov and Kuznetsova 2010). Although several authors have suggested the loss of TTAGG repeat in all 

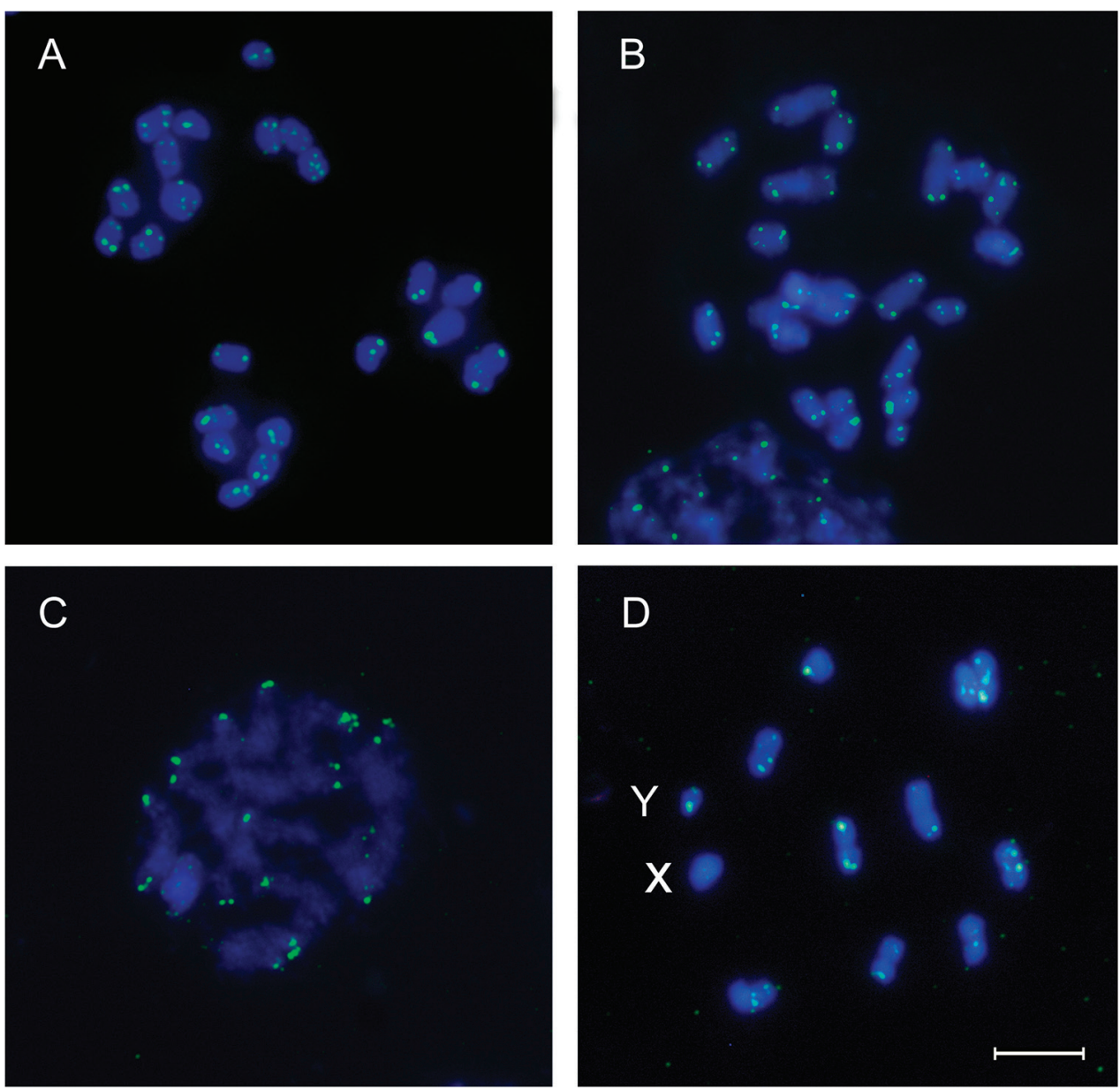

Figure I. Fluorescence in situ hybridization with (TTAGG) ${ }_{n}$ telomeric probe (green signals) on mitotic and meiotic chromosomes (counterstained with DAPI in blue) of four Triatominae species. A Triatoma infestans $(2 \mathrm{n}=22)$, spermatogonial prometaphase $\mathbf{B}$ Triatoma dimidiata $(2 \mathrm{n}=23)$, spermatogonial prometaphase $\mathbf{C}$ Dipetalogaster maxima $(2 \mathrm{n}=22)$, pachytene stage $\mathbf{D}$ Rhodnius prolixus $(2 \mathrm{n}=22)$, first meiotic division showing 10 bivalents and two sex chromosomes (X and Y). Scale bar: $5 \mu \mathrm{m}$.

Cimicomorpha species (Grozeva et al. 2015, Mason et al. 2016), the results presented here clearly contradict this hypothesis. According to the most comprehensive phylogeny of assassin bugs, the subfamily Triatominae is the youngest within Reduviidae, having evolved in the Oligocene, approximately 32 million years ago (24-38 Ma) (Hwang and Weirauch 2012). Whereas, a new acquisition of telomeric repeat in this recent evolutionary group seems unlikely, probably this lack of detection in Cimicomorpha and Pentatomomorpha is due to a methodological problem of the telomeric probe rather than a loss process during their evolution. Detailed analyses of the genomes repetitive fraction as well as exhaustive bioinformatics search on genomic databases might clarify the existence of these repeat sequences in other heteropteran groups. 


\section{Acknowledgments}

This study was supported by project grants (No. 370) from the "Comisión Sectorial de Investigación Científica" (CSIC-Udelar-Uruguay), Programa de Desarrollo de las Ciencias Básicas (PEDECIBA Uruguay), Agencia Nacional de Investigación e Innovación (ANII, Uruguay) and by the "Consejería de Innovación, Ciencia y Empresa de la Junta de Andalucía", sponsor of Program of Academic Mobility of AUIP (IberoAmerican University Postgraduate Association) for S.P. and F.P. This work was also supported by the Spanish Junta de Andalucía (through the program "Ayudas a Grupos de Investigación", Group BIO220). This paper is included in the Ph.D. Thesis of Sebastián Pita (Udelar-University of Jaén), partially funded by a grant from the University of Jaén through the program "Ayudas para la realización de tesis doctoral en régimen de cotutela”.

\section{References}

Altemose N, Miga KH, Maggioni M, Willard HF (2014) Genomic characterization of large heterochromatic gaps in the human genome assembly. PLoS Computational Biology 10(5): e1003628. doi: 10.1371/journal.pcbi.1003628

Frydrychová R, Marec F (2002) Repeated losses of TTAGG telomere repeats in evolution of beetles (Coleoptera). Genetica 115: 179-187. doi: 10.1023/A:1020175912128

Frydrychová R, Grossmann P, Trubač P, Vítková M, Marec F (2004) Phylogenetic distribution of TTAGG telomeric repeats in insects. Genome 47: 163-178. doi: 10.1007/s10577-005-7721-0 Gokhman VE, Anokhin BA, Kuznetsova VG (2014) Distribution of 18S rDNA sites and absence of the canonical TTAGG insect telomeric repeat in parasitoid Hymenoptera. Genetica 142: 317-322. doi: 10.1007/s10709-014-9776-3

Golub NV, Kuznetsova VG, Rakitov RA (2014) First karyotype data on the family Myerslopiidae (Hemiptera, Auchenorrhyncha, Cicadomorpha). Comparative Cytogenetics 8: 293-300. doi: 10.3897/CompCytogen.v8i4.8813

Golub NV, Golub VB, Kuznetsova VG (2015) Variability of 18rDNA loci in four lace bug species (Hemiptera, Tingidae) with the same chromosome number. Comparative Cytogenetics 9: 513-522. doi: 10.3897/CompCytogen.v9i4.5376

Grozeva S, Kuznetsova VG, Anokhin BA (2011) Karyotypes, male meiosis and comparative FISH mapping of $18 \mathrm{~S}$ ribosomal DNA and telomeric (TTAGG)n repeat in eight species of true bugs (Hemiptera, Heteroptera). Comparative Cytogenetics 5: 97-116. doi: 10.3897/ CompCytogen.v5i4.2307

Grozeva S, Anokhin BA, Kuznetsova VG (2015) Bedbugs (Hemiptera). In: Sharakhov IV (Ed.) Protocols for Cytogenetic Mapping of Arthropod Genomes, Chapter 8. CRC Press, Boca Raton, 285-326.

Hwang WS, Weirauch C (2012) Evolutionary history of assassin bugs (Insecta: Hemiptera: Reduviidae): insights from divergence dating and ancestral state reconstruction. PLoS ONE 7: e45523. doi: 10.1371/journal.pone.0045523 
IJdo JW, Wells RA, Baldini A, Reeders ST (1991) Improved telomere detection using a telomere repeat probe (TTAGGG)n generated by PCR. Nucleic Acids Research 19: 4780. doi: $10.1093 /$ nar/19.17.4780

Korandová M, Krůček T, Vrbová K, Čapková Frydrychová R (2014) Distribution of TTAGGspecific telomerase activity in insects. Chromosome Research 22: 495-503. doi: 10.1007/ s10577-014-9436-6

Kuznetsova VG, Grozeva SM, Nokkala S, Nokkala C (2011) Cytogenetics of the true bug infraorder Cimicomorpha (Hemiptera, Heteroptera): a review. Zookeys 154: 31-70. doi: 10.3897/zookeys.154.1953

Kuznetsova VG, Grozeva S, Anokhin B (2012) The first finding of (TTAGG)n telomeric repeat in chromosomes of true bugs (Heteroptera, Belostomatidae). Comparative Cytogenetics 6: 341-346. doi: 10.3897/CompCytogen.v6i4.4058

Kuznetsova VG, Maryańska-Nadachowska A, Anokhin B, Aguin-Pombo D (2015a) Evidence for TTAGG telomere repeats and rRNA gene clusters in leafhoppers of the genus Alebra (Hemiptera: Auchenorrhyncha: Cicadellidae). European Journal of Entomology 112: 207214. doi: 10.14411/eje.2015.045

Kuznetsova VG, Grozeva SM, Hartung V, Anokhin BA (2015b) First evidence for (TTAGG) $\mathrm{n}$ telomere sequence and sex chromosome post-reduction in Coleorrhyncha (Insecta, Hemiptera). Comparative Cytogenetics 9: 523-532. doi: 10.3897/CompCytogen.v9i4.5609

Lorite P, Carrillo JA, Palomeque T (2002) Conservation of (TTAGG)n telomeric sequences among ants (Hymenoptera, Formicidae). Journal of Heredity 93: 282-285. doi: 10.1093/ jhered/93.4.282

Lukhtanov VA, Kuznetsova VG (2010) What genes and chromosomes say about the origin and evolution of insects and other arthropods. Russian Journal of Genetics 46: 1115-1121. doi: 10.1134/S1022795410090279

Mandrioli M (2002) Cytogenetic characterization of telomeres in the holocentric chromosomes of the lepidopteran Mamestra brassicae. Chromosome Research 10: 279-286. doi: 10.1023/A:1016515607278

Maryańska-Nadachowska A, Kuznetsova VG, Karamysheva TV (2013) Chromosomal location of rDNA clusters and TTAGG telomeric repeats in eight species of the spittlebug genus Philaenus (Hemiptera: Auchenorrhyncha: Aphrophoridae). European Journal of Entomology 110: 411-418. doi: 10.14411/eje.2013.055

Mason JM, Čapková Frydrychová R, Biessmann H (2008) Drosophila telomeres: an exception providing new insights. BioEssays 30: 25-37. doi: 10.1002/bies.20688

Mason JM, Randall TA, Frydrychová RC (2016) Telomerase lost? Chromosoma 125: 65-73. doi: $10.1007 / \mathrm{s} 00412-015-0528-7$

Mesquita RD, Vionette-Amaral RJ, Lowenberger C, Rivera-Pomar R, Monteiro FA, Minx P, Spieth J, Carvalho AB, Panzera F, Lawson D, Torres AQ, Ribeiro JM, Sorgine MH, Waterhouse RM, Montague MJ, Abad-Franch F, Alves-Bezerra M, Amaral LR, Araujo HM, Araujo RN, Aravind L, Atella GC, Azambuja P, Berni M, Bittencourt-Cunha PR, Braz GR, Calderón-Fernández G, Carareto CM, Christensen MB, Costa IR, Costa SG, Dansa M, Daumas-Filho CR, De-Paula IF, Dias FA, Dimopoulos G, Emrich SJ, EspondaBehrens N, Fampa P, Fernandez-Medina RD, da Fonseca RN, Fontenele M, Fronick C, 
Fulton LA, Gandara AC, Garcia ES, Genta FA, Giraldo-Calderón GI, Gomes B, Gondim KC, Granzotto A, Guarneri AA, Guigó R, Harry M, Hughes DS, Jablonka W, JacquinJoly E, Juárez MP, Koerich LB, Lange AB, Latorre-Estivalis JM, Lavore A, Lawrence GG, Lazoski C, Lazzari CR, Lopes RR, Lorenzo MG, Lugon MD, Majerowicz D, Marcet PL, Mariotti M, Masuda H, Megy K, Melo AC, Missirlis F, Mota T, Noriega FG, Nouzova M, Nunes RD, Oliveira RL, Oliveira-Silveira G, Ons S, Orchard I, Pagola L, Paiva-Silva GO, Pascual A, Pavan MG, Pedrini N, Peixoto AA, Pereira MH, Pike A, Polycarpo C, Prosdocimi F, Ribeiro-Rodrigues R, Robertson HM, Salerno AP, Salmon D, Santesmasses D, Schama R, Seabra-Junior ES, Silva-Cardoso L, Silva-Neto MA, Souza-Gomes M, Sterkel M, Taracena ML, Tojo M, Tu ZJ, Tubio JM, Ursic-Bedoya R, Venancio TM, Walter-Nuno AB, Wilson D, Warren WC, Wilson RK, Huebner E, Dotson EM, Oliveira PL (2015) Genome of Rhodnius prolixus, an insect vector of Chagas disease, reveals unique adaptations to hematophagy and parasite infection. Proceedings of the National Academy of Sciences of the United States of America 112: 14936-14941. doi: 10.1073/ pnas. 1506226112

Meyne J, Ratliff RL, Moyzis RK (1989) Conservation of the human telomere sequence (TTAGGG)n among vertebrates. Proceedings of the National Academy of Sciences of the United States of America 86: 7049-7053. doi: 10.1073/pnas.86.18.7049

Mohan KN, Rani BS, Kulashreshta PS, Kadandale JS (2011) Characterization of TTAGG telomeric repeats, their interstitial occurrence and constitutively active telomerase in the mealybug Planococcus lilacinus (Homoptera; Coccoidea). Chromosoma 120: 165-175. doi: 10.1007/s00412-010-0299-0

Monti V, Giusti G, Bizzaro D, Manicardi GC, Mandrioli M (2011) Presence of a functional (TTAGG)n telomere-telomerase system in aphids. Chromosome Research 19: 625-633. doi: 10.1007/s10577-011-9222-7

Mora P, Vela J, Sanllorente O, Palomeque T, Lorite P (2015) Molecular cytogenetic studies in the ladybird beetle Henosepilachna argus (Coleoptera, Coccinellidae, Epilachninae). Comparative Cytogenetics 9: 423-434. doi: 10.3897/CompCytogen.v9i3.5263

Mravinac B, Meštrović N, Čavrak VV, Plohl M (2011) TCAGG, an alternative telomeric sequence in insects. Chromosoma 120: 367-376. doi: 10.1007/s00412-011-0317-x

Novák P, Neumann P, Macas J (2010) Graph-based clustering and characterization of repetitive sequences in next-generation sequencing data. BMC Bioinformatics 11: 378. doi: 10.1186/1471-2105-11-378

Novák P, Neumann P, Pech J, Steinhaisl J, Macas J (2013) RepeatExplorer: A Galaxy-based web server for genome-wide characterization of eukaryotic repetitive elements from next-generation sequence reads. Bioinformatics 29: 792-793. doi: 10.1093/bioinformatics/btt054

Novotná J, Havelka J, Starý P, Koutecký P, Vítková M (2011) Karyotype analysis of the Russian wheat aphid, Diuraphis noxia (Kurdjumov) (Hemiptera: Aphididae) reveals a large X chromosome with rRNA and histone gene families. Genetica 139: 281-289. doi: 10.1007/ s10709-011-9546-4

Okazaki S, Tsuchida K, Maekawa H, Ishikawa H, Fujiwara H (1993) Identification of a pentanucleotide telomeric sequence, (TTAGG)n, in the silkworm Bombyx mori and in other insects. Molecular and Cellular Biology 13: 1424-1432. doi: 10.1128/MCB.13.3.1424 
Panzera F, Ferrandis I, Ramsey J, Salazar-Schettino PM, Cabrera M, Monroy C, Bargues MD, Mas-Coma S, O’Connor JE, Angulo VM, Jaramillo N, Pérez R (2007) Genome size determination in Chagas disease transmitting bugs (Hemiptera-Triatominae) by flow cytometry. American Journal of Tropical Medicine, Hygiene 76: 516-521.

Panzera F, Pérez R, Panzera Y, Ferrandis I, Ferreiro MJ, Calleros L (2010) Cytogenetics and genome evolution in the subfamily Triatominae (Hemiptera, Reduviidae). Cytogenetic and Genome Research 128: 77-87. doi: 10.1159/000298824

Sahara K, Marec F, Traut W (1999) TTAGG telomeric repeats in chromosomes of some insects and other arthropods. Chromosome Research 7:449-460. doi: 10.1023/A:1009297729547

Richards S, Gibbs RA, Weinstock GM, Brown SJ, Denell R, Beeman RW, Gibbs R, Beeman RW, Brown SJ, Bucher G, Friedrich M, Grimmelikhuijzen CJ, Klingler M, Lorenzen M, Richards S, Roth S, Schröder R, Tautz D, Zdobnov EM, Muzny D, Gibbs RA, Weinstock GM, Attaway T, Bell S, Buhay CJ, Chandrabose MN, Chavez D, Clerk-Blankenburg KP, Cree A, Dao M, Davis C, Chacko J, Dinh H, Dugan-Rocha S, Fowler G, Garner TT, Garnes J, Gnirke A, Hawes A, Hernandez J, Hines S, Holder M, Hume J, Jhangiani SN, Joshi V, Khan ZM, Jackson L, Kovar C, Kowis A, Lee S, Lewis LR, Margolis J, Morgan M, Nazareth LV, Nguyen N, Okwuonu G, Parker D, Richards S, Ruiz SJ, Santibanez J, Savard J, Scherer SE, Schneider B, Sodergren E, Tautz D, Vattahil S, Villasana D, White CS, Wright R, Park Y, Beeman RW, Lord J, Oppert B, Lorenzen M, Brown S, Wang L, Savard J, Tautz D, Richards S, Weinstock G, Gibbs RA, Liu Y, Worley K, Weinstock G, Elsik CG, Reese JT, Elhaik E, Landan G, Graur D, Arensburger P, Atkinson P, Beeman RW, Beidler J, Brown SJ, Demuth JP, Drury DW, Du YZ, Fujiwara H, Lorenzen M, Maselli V, Osanai M, Park Y, Robertson HM, Tu Z, Wang JJ, Wang S, Richards S, Song H, Zhang L, Sodergren E, Werner D, Stanke M, Morgenstern B, Solovyev V, Kosarev P, Brown G, Chen HC, Ermolaeva O, Hlavina W, Kapustin Y, Kiryutin B, Kitts P, Maglott D, Pruitt K, Sapojnikov V, Souvorov A, Mackey AJ, Waterhouse RM, Wyder S, Zdobnov EM, Zdobnov EM, Wyder S, Kriventseva EV, Kadowaki T, Bork P, Aranda M, Bao R, Beermann A, Berns N, Bolognesi R, Bonneton F, Bopp D, Brown SJ, Bucher G, Butts T, Chaumot A, Denell RE, Ferrier DE, Friedrich M, Gordon CM, Jindra M, Klingler M, Lan Q, Lattorff HM, Laudet V, von Levetsow C, Liu Z, Lutz R, Lynch JA, da Fonseca RN, Posnien N, Reuter R, Roth S, Savard J, Schinko JB, Schmitt C, Schoppmeier M, Schröder R, Shippy TD, Simonnet F, Marques-Souza H, Tautz D, Tomoyasu Y, Trauner J, Van der Zee M, Vervoort M, Wittkopp N, Wimmer EA, Yang X, Jones AK, Sattelle DB, Ebert PR, Nelson D, Scott JG, Beeman RW, Muthukrishnan S, Kramer KJ, Arakane Y, Beeman RW, Zhu Q, Hogenkamp D, Dixit R, Oppert B, Jiang H, Zou Z, Marshall J, Elpidina E, Vinokurov K, Oppert C, Zou Z, Evans J, Lu Z, Zhao P, Sumathipala N, Altincicek B, Vilcinskas A, Williams M, Hultmark D, Hetru C, Jiang H, Grimmelikhuijzen CJ, Hauser F, Cazzamali G, Williamson M, Park Y, Li B, Tanaka Y, Predel R, Neupert S, Schachtner J, Verleyen P, Raible F, Bork P, Friedrich M, Walden KK, Robertson HM, Angeli S, Forêt S, Bucher G, Schuetz S, Maleszka R, Wimmer EA, Beeman RW, Lorenzen M, Tomoyasu Y, Miller SC, Grossmann D, Bucher G (2008) The genome of the model beetle and pest Tribolium castaneum. Nature 452: 949-955. doi: 10.1038/nature06784 
Vítková M, Král J, Traut W, Zrzavý J, Marec F (2005) The evolutionary origin of insect telomeric repeats, (TTAGG)n. Chromosome Research 13: 145-156. doi: 10.1007/s10577005-7721-0

Walter MF, Bozorgnia L, Maheshwari A, Biessmann H (2001) The rate of terminal nucleotide loss from a telomere of the mosquito Anopheles gambiae. Insect Molecular Biology 10: $105-110$.

Watson JM, Riha K (2010) Comparative biology of telomeres: Where plants stand. Febs Letters 584: 3752-3759. doi: 10.1046/j.1365-2583.2001.00245.x

Weirauch C, Schuh RT (2011) Systematics and evolution of Heteroptera: 25 years of progress. Annual Review of Entomology 56: 487-510. doi: 10.1146/annurev-ento-120709-144833

Zakian VA (1995) Telomeres: beginning to understand the end. Science 270: 1601-1607. doi: $10.1126 /$ science. 270.5242 .1601 